\title{
Recognition of the anatomical structure of the atrioventricular node: Connection between the retroaortic node and the compact node
}

\author{
Fan Wang ${ }^{1}$, Lulu Zhang ${ }^{1}$, Wei Meng ${ }^{1}$, Bin Zhu ${ }^{1}$, Shufeng $\mathrm{Li}^{1}$, Qiuwen $\mathrm{Wu}^{2}$, and shuo \\ zhang ${ }^{1}$ \\ ${ }^{1}$ Second Affiliated Hospital of Harbin Medical University \\ ${ }^{2}$ Harbin Medical University Second Affiliated Hospital Department of Cardiology
}

December 16, 2020

\begin{abstract}
Introduction: The complex electrophysiological phenomena related to the atrioventricular node (AVN) are due to its complex anatomical structures. Aside from the inferior nodal extension (INE), other node-like tissues, such as the retroaortic node (RN), have been less described and may also share the mechanism of normal conduction and abnormal conduction in AVN re-entrant tachycardia (AVNRT). Methods: High-density sections of the entire AVN were obtained from rats and rabbits. Fibrosis was analyzed by Masson's trichrome staining. Connexin (Cx43, Cx40, and Cx45) and ion channel (Nav1.5, Cav3.1, and HCN4) proteins were immunohistochemically labeled for the analysis of tissue features. Three-dimensional (3D) reconstruction of the AV junction was performed to clarify the relationships among different structures. Results: The RN expressed the same connexin isoforms as the compact node $(\mathrm{CN})$ and INE. Nav1.5 labeling was present at a low level in the CN, RN and INE, where Cav3.1 and HCN4 were expressed. The CN connected with the RN in a narrow strip pattern at the level of the start of the CN. The RN presented as a shuttle shape and was the only tissue directly connected with the atrium in the anterior septum. Conclusion: The RN connects with the AVN anatomically, suggesting that there is direct electrical conduction between them. The entrance of the atria into the AVN is the distal part of the RN, which may form the fast pathway of the AVN.
\end{abstract}

\section{Introduction}

The atrioventricular node (AVN) lies within the triangle of Koch and is bound by the coronary sinus, tendon of Todaro and tricuspid valve. The AVN functions by conducting action potentials at an appropriate conduction velocity from the atria to the ventricles. The AVN also acts as a backup pacemaker in the case of sinus node failure. On the other hand, the AVN is part of the underlying circuitry that causes AVN reentrant tachycardia (AVNRT). ${ }^{1}$ Inferior nodal extensions (INEs) are part of the AVN,${ }^{1-5}$ and characteristics of these structures are conserved among various species, including humans, ${ }^{2}$ rats $^{3}{ }^{3}$ mice, ${ }^{4}$ rabbits ${ }^{5}$ and dogs. Moreover, it is thought that these extensions may be involved in slow-pathway conduction and are part of the underlying circuitry that causes AVNRT, which is supported by electrophysiological studies. ${ }^{6,7}$ The use of catheter or surgical techniques targeting injuries in this region is thought to be the best method for curing these forms of tachycardia. ${ }^{7}$ However, there are still some electrophysiological phenomena that cannot be explained by relying only on these anatomical structures, as the exact circuitry responsible for reentrant tachycardias in different types of AVNRT remains unknown. Complex anatomical structures and functional longitudinal dissociations are considered important in understanding the behavior of these tachycardias. ${ }^{1}$ Other conduction tissues may also participate in the real mechanism of AVNRT. The retroaortic node (RN) has been described as a heap of node-like tissue located on the right side of the atrium neighboring the aorta. ${ }^{8}$ Previous studies have described the anatomical features of this region and its relationship with the compact node $(\mathrm{CN})$ in little detail. The potential electrophysiological function of these node-like tissues also remains unknown. 
The aim of this study was to comprehensively analyze the detailed anatomical structure and histological features of this node-like tissue and better understand its electrophysiological behavior in normal AV conduction and AVNRT.

\section{Methods}

Animals and serial sectioning of the AVN

Adult Sprague-Dawley (SD) rats $(\mathrm{n}=6)$ and adult New Zealand White rabbits $(\mathrm{n}=5)$ were used. All animals were obtained from the Laboratory Animal Science Department, the Second Affiliated Hospital of Harbin Medical University, Heilongjiang, PR China. All experimental animal procedures were approved by the Local Ethics Committee of Harbin Medical University Animal Care and Use. Briefly, animals were euthanized by cervical dislocation. The heart was removed from each animal, and the AVN region (Figure 1A) was dissected on ice. The AVN region was embedded in optimal cutting temperature (OCT) compound. Four marker points were drawn on the mold in OCT to mark the position of the AVN before it was snap-frozen by liquid nitrogen. Serial cryosections $(6-7 \mu \mathrm{m})$ were obtained from the AVN tissue by placing the microtome blade at an angle of $20-30^{\circ}$ to the vertical axis (Figure 1B), allowing maximum coverage of the atrium as well as visualization of the His bundle. Once typical characteristics of the penetrating bundle (PB) were identified by Masson's trichrome staining, serial sections were obtained and grouped in preparation for subsequent staining. Sections were processed up to the level of the coronary sinus structure.

Histological staining

Masson's trichrome staining (Nanjing Jiancheng Bioengineering Institute) was performed on samples from the AVN region according to the manufacturer's instructions to assess fibrosis and delineate the initiation of the inferior region of the AV conduction system. Acetylcholinesterase (AChE) staining was also performed on adjacent sections as a secondary confirmation of the conduction system, as previously described. ${ }^{9}$

Immunofluorescence

Adjacent sections from each group were double-labeled with connexin 43 (Cx43) and HCN4 antibodies, connexin $40(\mathrm{Cx} 40)$ and connexin $45(\mathrm{Cx} 45)$ antibodies, and $\mathrm{Na}_{\mathrm{v}} 1.5$ and $\mathrm{Ca}_{\mathrm{v}} 3.1$ antibodies. Briefly, sections were fixed in $4 \%$ paraformaldehyde solution for 10-15 minutes, washed three times with PBS (pH 7.4), treated with $0.3 \%$ Triton X-100 and blocked with $5 \%$ normal donkey serum for one hour at room temperature. Sections were incubated with the following primary antibodies in blocking solution at $4^{\circ} \mathrm{C}$ overnight: mouse anti-Cx45 (1:100; Abcam Inc.), goat anti-Cx40 (1:100; Santa Cruz Biotechnology, Inc.), rabbit anti-Cx43 (1:100; Cell Signaling Technology, Inc.), rabbit anti-Na 1.5 (1:200; Alomone Labs, Inc.), rabbit anti-Ca 3.1 (1:200; Alomone Labs, Inc.) and goat anti-HCN4 (1:50; Santa Cruz Biotechnology, Inc.). Subsequently, sections were extensively washed in PBS and incubated in a diluted solution for one hour at room temperature with the following secondary antibodies: DyLight 594-conjugated donkey anti-mouse (1:100; EarthOx, San Francisco, CA, USA), DyLight 488-conjugated donkey anti-goat (1:100; EarthOx, San Francisco, CA, USA), DyLight 488-conjugated donkey anti-rabbit (1:100; EarthOx, San Francisco, CA, USA), DyLight 594-conjugated donkey anti-goat (1:100; EarthOx, San Francisco, CA, USA) and DyLight 594-conjugated donkey anti-rabbit (1:100; EarthOx, San Francisco, CA, USA). Nuclei were stained with Draq5 (1:10000; Cell Signaling Technology) within the secondary antibody incubation step. Sections were mounted in mounting medium and imaged using a fluorescence microscope (LEICA DM4000B). Signal intensity measurements were then obtained using Image-Pro Plus 6.0 software after background correction.

Three-dimensional (3D) reconstruction of the AV junction

All sections collected from 80 groups (320 sections) from one rat were used to reconstruct a 3D computational model of the anatomy of the AVN. 3D-DOCTOR (version 4.0; Able Software Corp.) and Adobe Photoshop were used to analyze the images and construct a 3D anatomical model of the AVN. There were four steps in this process. Step 1: Segmentation. Various tissue types could be identified in Masson's trichrome-stained sections: myocytes were stained purple, and connective tissues were stained blue. Further cell types were 
identified by merging adjacent immunofluorescence-labeled images with images from similarly grouped Masson's trichrome-stained sections using Adobe Photoshop, and these combinations included the following: (i) Cx43-negative, Cx40-negative, HCN4-positive and strongly Cx45-positive nodal tissues; (ii) Cx43-negative, Cx40-positive, HCN4-positive and strongly Cx45-positive His bundle tissues; and (iii) Cx43-positive ventricular and atrial muscles. Then, Adobe Photoshop was used to outline various cell types (carried out manually) on images obtained from Masson's trichrome-stained sections. A unique color was used to highlight each cell type. Finally, multicolor images were converted into grayscale images in preparation for 3D reconstruction. The result was a two-dimensional model of sections showing the distribution of myocytes, connective tissues and different cell types. Step 2: Alignment.Sections were aligned by some combination of translation, rotation and scaling, but the shape of the sections was left unchanged. The alignment of sections is crucial in developing a model. Tracts of the nodal tissue, tricuspid valve and bottom edge of the preparation were the main landmarks used to align the sections. Each image was aligned by 3D-DOCTOR and saved. The saved aligned images were used in preparation for the next step of 3D reconstruction. Step 3: Calibration . The voxel size (image resolution) must be provided to obtain a 3D image so that the $3 \mathrm{D}$ rendering has the correct scale in all three dimensions, enabling it to incorporate the reported measurements. For the voxel resolution, the $\mathrm{X}$ and $\mathrm{Y}$ values are equivalent to the size of one pixel in a slice, while the $\mathrm{Z}$ value is the slice thickness and distance between the centers of two neighboring slices in 3D space. A group at the same level of the tissues was created as an assumption; thus, the distance between two prepared grayscale images was $24 / 28 \mu \mathrm{m}\left(6 / 7 \mu \mathrm{m}^{*} 4\right)$. Volume correlation measurements were based on parameters obtained from the image and scale plate provided by the camera $(1 \mu \mathrm{m}=1.17$ unit). As a result, $\mathrm{Z}$ values $(28.08 / 32.76$ units $)$ were used as the thickness between the two groups of sections, which is an option for 3D reconstruction. Step 4: Model visualization. After creating an interactive segment, surface rendering was performed using 3D-DOCTOR. Although only two preparations were used to generate an anatomical model, we confirmed these anatomical findings in four other preparations.

\section{Statistical analysis}

Data are presented as the mean \pm standard error of the mean (SEM). Significant differences in the abundance of proteins in different tissues were identified using one-way ANOVA. Significant differences were determined when $P<0.05$. The intensity of $\mathrm{Na}_{\mathrm{v}} 1.5$ staining was analyzed in different areas of the AV junction, including $\mathrm{RN}, \mathrm{CN}, \mathrm{INE}$, atrial and ventricular tissues, using quantitative immunofluorescence staining analysis. The background level of staining in each tissue section can affect the results obtained from immunofluorescence staining; thus, the staining intensity of $\mathrm{Na}_{\mathrm{v}} 1.5$ in ventricular tissue was included and used as a reference in each section used for analysis.

\section{Results}

Location of the RN, CN and inferior extension

Serial sections (a total of 80 groups) were chosen from an adult rat and revealed the most integrated conduction system. The sequence of sections began from the anterior septum to the posterior septum. The structure of the RN roughly presented from the $1^{\text {st }}$ section to the $37^{\text {th }}$ group of sections neighboring the aorta above the His bundle. Further analysis was performed on the $13^{\text {th }}$ group of sections, shown in Figure 2A-D, which presented the main body of the $\mathrm{RN}$ and showed its positional relationship with the His bundle. Masson's trichrome staining showed that the PB tissue (light purple) was surrounded by fibrous tissue (blue) on the ventricular side (Figure 2A). AChE expression was positive on the atrial side over the tricuspid ring, suggesting the distribution of $\mathrm{RN}$ (Figure $2 \mathrm{~B}$, arrow). Figure $2 \mathrm{C}$ highlights the absence of $\mathrm{Cx} 43$ expression (green) in both the atrial and ventricular regions, while the expression of AChE and HCN4 was observed in $\mathrm{Cx} 43$-negative regions. Figure 2D shows that $\mathrm{Cx} 45$ expression was abundant in the $\mathrm{PB}$ and the areas where Cx43 expression was absent on the atrial side. Cx40 was expressed only in PB tissues.

The structure of the $\mathrm{CN}$ roughly presented from the $38^{\text {th }}$ section to $53^{\text {rd }}$ group of sections. Figure $2 \mathrm{E}-\mathrm{H}$ represents the $39^{\text {th }}$ group of serial sections at the level coincident with the CN. The CN is considered to be the start of the PB. ${ }^{10}$ Masson's trichrome staining showed the transit of all AVN tissues nearly to the 
atrial side (Figure 2E). AChE-positive staining was present mostly in the area of the CN, and little could be seen above the CN, which suggests the end of the RN (Figure $2 \mathrm{~F}$, arrow). At this level, $\mathrm{Cx} 43$ and HCN4 double-labeling showed the greatest absence of $\mathrm{Cx} 43$ expression limited to the AVN area where HCN4 expression was positive (Figure 2G). Little of the HCN4-positive area on the atrial side coincided with the AChE-positive site. As shown in Figure 2H, Cx45 was found to be abundant in the AVN, although there was slight cross-reactivity with the connective tissue on the ventricular side. In addition, a punctate distribution pattern of Cx40 expression showed a disappearing trend (elliptical area).

The structure of the inferior extension of the AVN roughly presented from $54^{\text {th }}$ group of sections to the $80^{\text {th }}$ group of sections. The 79th group of sections was near the coronary sinus level. Masson's trichrome staining showed the inferior extension of the AVN located on the atrial side above the tricuspid annules (Figure 2I). AChE staining was limited to the INE area (Figure 2J). The volume of the inferior extension region also decreased moving away from the $\mathrm{CN}$. Cx43 expression was absent in the inferior extension where HCN4 expression was positive (Figure $3 \mathrm{~K}$ ). Cx45 expression was relatively more abundant in the INE than in other atrial areas (Figure 3L, a slight fold in the section). No Cx40 expression was observed in the inferior extension. The inferior extension divided into right and left extensions at this level (Figure 3K).

Characteristics of RN and CN connection

The location of the main body of the $\mathrm{RN}$ was the same in our as in previous reports. ${ }^{3,8}$ Additional information we intended to acquire was the relationship between the RN and CN. Continuous sections at the level of the start of the $\mathrm{CN}$ and the end of the $\mathrm{RN}$ from another sample were chosen to show the structural features in this region. Part of the node-like tissue was separated from the $\mathrm{RN}$ and penetrated into the fibrous rings between the atrium and ventricle (Figure 3A1-3, dotted box). The following continuous sections (Figure 3B and $\mathrm{C}$, dotted box) showed that the node tissue descended into the CN. HCN4 labeling further suggested that the penetrated tissue was node-like tissue. As proven repeatedly by other samples in our study, the level of the start of the $\mathrm{CN}$ is a more complex region than previously described. First, it stretches downward forming the transitional area between the His bundle and AVN; second, it extends backward, forming the main body of the $\mathrm{CN}$; third, it accepts the insertion of the end of the RN.

Some of the samples suggested that the region connecting the RN and CN was also near the start of the tendon of Todaro (TT) (Figure 3D-F). Two narrow connections between the RN and CN at different levels near the TT region are shown in Figure 3D and E (dotted box), which suggests that there may be more than one connection between the RN and CN.

Fibrous tissue surrounding the $\mathrm{CN}$ at the level of almost the entire $\mathrm{CN}$ seems to have no direct connection with the atrium, while most of the $\mathrm{RN}$ was directly connected to the atrium, without surrounding fibrous tissue (Masson's staining, Figures 2A and 3F).

$3 \mathrm{D}$ reconstruction of the $\mathrm{AV}$ junction

A more direct view of the relationships among different structures could be achieved following the 3D reconstruction of the AV junction. In summary, atrial and ventricular tissues (Cx43-positive and HCN4negative tissues), nodal tissue (Cx43-negative; HCN4-positive; $\mathrm{Cx} 40$-negative and strongly $\mathrm{Cx} 45$-positive tissue), PB tissue (Cx43-negative; HCN4-positive; Cx40-positive and strongly $\mathrm{Cx} 45$-positive tissue) and connective tissue (Masson's trichrome-stained tissue, blue) were present at the AV junction. Figure 4A summarizes the distribution of cell types in all 80 levels that were studied (images are prior to alignment), wherein only the tract of the nodal tissue and nearby tissue is shown. The nodal tissue includes the CN, inferior extension and RN (bright yellow), Cx40-positive tissue of the PB (red), atrial and ventricular muscle (sandy), and connective tissue (light blue), as shown in Figure 4 (left). These images of serial sections showed changing trends in the distribution, location and volume of cell types at the AV junction. The sections shown in Figure 4 (left) were used to generate a 3D anatomical model of the AVN.

Various views of the model are shown in Figure 4 (right) (atria and ventricles were removed to highlight junctional tissues). Based on the patterns of connexin and HCN4 expression, we propose that the anterior 
extension, $\mathrm{CN}$ and inferior extension contain the same cell types. The positional and volumetric relationships among them are shown in Figure 4 (right). The inferior extension gradually decreased in volume moving away from the $\mathrm{CN}$, with no obvious boundary with the CN. However, the shape of the RN was similar to a shuttle. Its connection with the $\mathrm{CN}$ was narrow. It expanded, with an increasing volume moving away from the $\mathrm{CN}$, which then decreased again at the anterior septum. The volume of the PB decreased as the level increased and disappeared into the $\mathrm{CN}$ (red part of the model).

Histological characteristics of the AV junction at different levels

Except for the connexins that dominated the electronic conduction of the heart, as labeled above, three main ion channels (Nav1.5, Cav3.1 and HCN4) participating in the depolarization of myocardial cells were immunohistochemically labeled to investigate the conduction ability in different structures. ${ }^{3,11,12}$ Figure 5A shows that Nav1.5 expression was abundant in the atrial and ventricular muscles and weak in the PB and $\mathrm{RN}$, while Cav3.1 was mainly expressed in the PB and RN. Figure $5 \mathrm{~B}$ and $\mathrm{C}$ shows that the $\mathrm{CN}$ and INE also weakly expressed $\mathrm{Na}_{\mathrm{v}} 1.5$ but positively expressed $\mathrm{Ca}_{\mathrm{v}} 3.1$. Further analysis of $\mathrm{Na}_{\mathrm{v}} 1.5$ expression via immunofluorescence microscopy showed that the RN, CN and INE all presented with significantly lower Nav1.5 expression $(\mathrm{P}<0.001)$. No significant differences in the expression levels were observed among the $\mathrm{RN}$, CN and INE $(\mathrm{P}=0.104)$, but there was a trend of slightly higher expression of $\mathrm{Na}_{\mathrm{v}} 1.5$ in the $\mathrm{RN}$ than in the other two regions.

\section{Discussion}

Our study is the first to describe the morphological characteristics of the RN and its relationship with the CN in detail. Serial histological and immunohistochemical labeling combined with 3D reconstruction provided an advantage in identifying structural features in this region. The results show a direct connection between the $\mathrm{CN}$ and RN. Cardiac myocytes located in the INE were first described by Anderson and colleagues as part of a continuous structure around the tricuspid valve. ${ }^{5}$ A separate study reported that the right nodal extension continues around the tricuspid valve annulus as the right AV ring forms, whereas the left nodal extension continues around the mitral valve annulus as the left AV ring forms. The right and left AV rings loop around the two valves and meet again to form the RN. ${ }^{8}$ Previous studies have described the anatomical location of this region in little detail, and its relationship within the $\mathrm{CN}$ is even less clear. Due to the high-density continuous sections, multiple-staining method and immunolabelling, our study reveals that the CN and the $\mathrm{RN}$ connect at the start of the $\mathrm{CN}$ and seem to constitute the only pathway connecting the atrium to the conduction tissue in the anterior septum. Previous studies have neglected this connection, which may have been caused by the insufficient density of continuous sections in this area and the subtle, narrow connection pattern. The proven connection between the $\mathrm{CN}$ and the RN provides a new substrate that may further explain the complex electrophysiological phenomena that occur in the AVN area.

Conduction characteristics affected by histological features in the AV junction

The principal connexin in the working atrial and ventricular myocardium is $\mathrm{Cx} 43$, with medium conductance. ${ }^{2,4,6}$ The $\mathrm{CN}$ shows low expression levels of $\mathrm{Cx} 43$ in humans and animals. ${ }^{4,13}$ However, Cx40 expression (at both the mRNA and protein levels) is high in the CN and PB, where it is implicated in facilitating fast conduction in humans and rats. ${ }^{3,10} \mathrm{Cx} 45$ is the predominant connexin expressed in the mammalian AVN. ${ }^{14}$ Thus, connexin expression could be used as a marker to recognize different conduction cell types. Moreover, connexins dominate electronic conduction, which provides approximately $30 \%$ of the conduction velocity. Another approximately $70 \%$ is dominated by ion channels. ${ }^{15}$ The RN that we describe in this study displayed the same pattern of connexin isoform expression as the CN and INE. Immunohistochemical labeling of the $\mathrm{Na}_{\mathrm{v}} 1.5, \mathrm{Ca}_{\mathrm{v}} 3.1$ and $\mathrm{HCN} 4$ channels showed nearly equal expression of these ion channels in the CN, INE and RN (Figure 5D), indicating that these areas have highly uniform and conserved conduction properties. These data suggest that the RN and its connection with the CN may have the same conduction properties as the $\mathrm{CN}$ and INE, exhibiting relatively slow conduction.

Fast AV nodal pathway and structural anatomical features of the AVN 
Single-cell features (which include the gene expression patterns of ion channels and connexins of single cells) and anatomical structures are equally important in providing information on conduction velocity. ${ }^{15}$ Thus, the focus of our study was to clarify the conduction relationships among the CN, INE, RN and atrium. Identifying the entrance of the atria into the AVN is very important in understanding the mechanisms driving normal and abnormal AV conduction as well as some arrhythmias associated with the AVN, such as AVNRT. Fibrous tissues separate the cardiac muscle to prevent electrical conduction and provide a structural basis for the direction of conduction. Our results show a striking amount of connective tissue surrounding the CN at the level of almost the entire $\mathrm{CN}$ and extending to the forefront until the start of the CN, suggesting that there was no obvious direct connection between the atrium and most of the CN. The end part of the RN (the part connecting with the $\mathrm{CN}$ ) seemed to be the only pathway connecting the atrium to the conduction tissue in the anterior septum (Figure 3). Because almost all of the retroaortic area presents a large connection with the atrial muscle, without surrounding fibrous tissues, this region is situated in an area that would more readily accept an impulse from the sinus node than the CN and INE. In a study by the M. Jackman team, ${ }^{16}$ the site of earliest atrial activation was described as being located posterior to (behind) the TT, outside the triangle of Koch, which coincides with our anatomical results. The region connecting the $\mathrm{RN}$ and $\mathrm{CN}$ was also near the start of the TT. The start of the CN was located at the apex of the Koch triangle, where the TT started on the atrial side (Figure 3). In some studies, ${ }^{17,18}$ early activation of the left side of the septum during AVNRT could be accounted for by the dispersed distribution of the RN in the atrial septum, containing the left side of the septum (Figure 2C). The descriptions of the histological characteristics and relationships of transitional tissue near the AV junction with the $\mathrm{CN}$ in previous studies have been confusing. The morphological features of transitional tissue cells have been described as between those of atrial cells and AV nodal cells, with low Cx43 or Nav1.5 expression. ${ }^{6,12}$ Some studies have suggested that the transitional cells above the CN may constitute the fast pathway entering the CN. ${ }^{6,12}$ In our study, we found that fibrous tissue surrounding the $\mathrm{CN}$ at the level of almost the entire $\mathrm{CN}$ seems unlikely to facilitate conduction in this direction, and the entrance of the AVN is more likely in the more forward position where the distal part of the $\mathrm{RN}$ is without surrounding fibrous tissue. Meanwhile, Antz et al. ${ }^{19}$ described the transitional cells crossing the TT toward the AV node. Microelectrode studies in rabbit hearts have suggested that these anterior transitional cells insert into the AV bundle relatively close to the central fibrous body ${ }^{20}$ The anatomical location of the transitional cells observed in these studies as the connection approaches the TT and inserts into the common AV bundle is similar to our description of the connection between the RN and $\mathrm{CN}$. As proven by the histological labeling of serial sections in our study, these tissues actually are node-like tissues in the continuation of the RN. In conclusion, the more accurate entrance of the atria into the AVN is more likely the small end part of the $\mathrm{RN}$, which could be proven by accurate patch-clamping techniques in the future.

AVNRT and anatomical structural features of the AV node

We consider the structure we described above to be the fast pathway of the AVN and to participate in the slow-fast type of AVNRT. During AVNRT, the earliest action site is not always as concentrated as that in atrial tachycardias (ATs), ${ }^{18}$ which could be explained by the dispersed connection pattern between the RN and atrium providing multiple exits in the retrograde region (Figure 6). Retrograde atrial activation most often occurs on the left (up to 53\%) rather than on the right side of the septum during AVNRT mapping, ${ }^{17}$ which could also be explained by the dispersed distribution of the RN in the atrial septum mentioned above. A study of isolated perfused canine hearts found that an anterograde conduction block in the fast pathway occurred near the TT. As the atrial pacing rate was increased, the block occurred at progressively more proximal locations, that is, closer to the anterior limbus of the fossa ovalis and further from the TT. ${ }^{19}$ This corresponds to the electrophysiological characteristics of node-like tissue, which we considered part of the RN. The site of the anterograde block in the fast pathway, outside the triangle of Koch, may prevent retrograde invasion of the slow pathway, and this may facilitate the initiation of "AV nodal" reentry. While the volume of the $\mathrm{CN}$ is not large at all, the connection with the $\mathrm{RN}$ is rather narrow, and the rate dependence of conduction slowing and blocking occurs at this natural isthmus in the slow-fast type of AVNRT (Figure 6).

While high-density sectioning and 3D reconstruction of the whole AV junction were used, it seems that the 
anatomical structure of the $\mathrm{RN}$ region is not related to the electrophysiological phenomena of other types of AVNRT described in previous studies.

\section{Conclusion}

We conclude that the RN connects with the AVN, which suggests direct electrical conduction between them. The entrance of the atria into the AVN is the distal part of the RN. The fast pathway of the AVN is strongly suggested by the structure of the RN and its extension. The level of the start of the CN is a more complex region than previously described. First, it stretches downward, forming the transitional area between the His bundle and AVN; second, it extends backward, forming the main body of the CN; and third, it accepts the insertion of the end of the RN.

\section{Acknowledgments}

No

\section{References}

1. Katritsis DG, Becker A. The atrioventricular nodal reentrant tachycardia circuit: a proposal. Heart Rhythm 2007;4:1354-1360.

2. Becker AE, Anderson RH. Morphology of the human atrioventricular junctional area. In: Wellens HJJ, Lie KI, Janse MJ, eds. The Conduction System of the Heart: Structure, Function and Clinical Implications. Leiden: HE Stenfert Kroese BV; 1978:263-286.

3. Yoo S, Dobrzynski H, Fedorov VV, et al. Localization of $\mathrm{Na}+$ channel isoforms at the atrioventricular junction and atrioventricular node in the rat. Circulation 2006;114:1360-1371.

4. Aanhaanen WTJ, Mommersteeg MTM, Norden J, et al. Developmental origin, growth, and threedimensional architecture of the atrioventricular conduction axis of the mouse heart. Circ Res 2010;107:728736 .

5. Anderson RH. The disposition and innervation of atrioventricular ring specialized tissue in rats and rabbits. J Anat 1972;113:197-211.

6. Li J, Greener ID, Inada S, et al. Computer three-dimensional reconstruction of the atrioventricular node. Circ Res 2008;102:975-985.

7. Lockwood DJ, Nakagawa H, Jackman WM. Electrophysiologic characteristics of atrioventricular nodal reentrant tachycardia: implications for reentrant circuits. In: Zipes DP, Jalife J, eds. Cardiac Electrophysiology: From Cell to Bedside. $5^{\text {th }}$ ed. Philadelphia: Saunders Elsevier; 2018:615-646.

8. Yanni J, Boyett MR, Anderson RH, Dobrzynski H. The extent of the specialized atrioventricular ring tissues. Heart Rhythm 2009;6:672-680.

9. El-Badawi A, Schenk EA. Histochemical methods for separate, consecutive and simultaneous demonstration of acetylcholinesterase and norepinephrine in cryostat sections. J Histochem Cytochem 1967;15:580-588.

10. Greener ID, Monfredi O, Inada S, et al. Molecular architecture of the human specialised atrioventricular conduction axis. J Mol Cell Cardiol 2011;50:642-651.

11. Liang X, Wang G, Lin L, et al. HCN4 dynamically marks the first heart field and conduction system precursors. Circ Res 2013;113:399-407.

12. Dobrzynski H, Nikolski VP, Sambelashvili AT, et al. Site of origin and molecular substrate of atrioventricular junctional rhythm in the rabbit heart. Circ Res 2003;93:1102-1110.

13. Hucker WJ, McCain ML, Laughner JI, Iaizzo PA, Efimov IR. Connexin 43 expression delineates two discrete pathways in the human atrioventricular junction. Anat Rec 2008;291:204-215. 
14. Coppen SR, Severs NJ, Gourdie RG. Connexin45 (?6) expression delineates an extended conduction system in the embryonic and mature rodent heart. Dev Genet 1999;24:82-90.

15. Smaill BH, Zhao J, Trew ML. Three-dimensional impulse propagation in myocardium: arrhythmogenic mechanisms at the tissue level. Circ Res 2013;112:834-848.

16. Otomo K, Antz M, Beckman KJ, et al. Left atrial activation precedes right atrial activation in Slow/Fast atrioventricular nodal reentrant tachycardia. Circulation 1996;94:3993-3993.

17. Hwang C, Martin DJ, Goodman JS, et al. Atypical atrioventricular node reciprocating tachycardia masquerading as tachycardia using a left-sided accessory pathway. J Am Coll Cardiol 1997;30:218-225.

18. Katritsis DG, Ellenbogen KA, Becker AE. Atrial activation during atrioventricular nodal reentrant tachycardia: studies on retrograde fast pathway conduction. Heart Rhythm 2006;3:993-1000.

19. Antz M, Scherlag BJ, Patterson E, et al. Electrophysiology of the right anterior approach to the atrioventricular node: studies in vivo and in the isolated perfused dog heart. Journal of Cardiovascular Electrophysiology 1997;8:47-61.

20. Patterson E, Scherlag BJ. Fast pathway-his bundle connections in the rabbit heart. Journal of Interventional Cardiac Electrophysiology 2004;10:121-129.

\section{Figure legends}

Figure 1. (A) Schematic of a heart with a window cut into the right atrium to visualize the location of the atrioventricular node (AVN). (B) Image of the AVN preparation used for constructing the model, the location of sections and the angle of the knife.

Figure 2. The $13^{\text {th }}, 39^{\text {th }}$, and $79^{\text {th }}$ groups of serial sections are shown. (A) Masson's trichrome-stained section. (B) AChE staining: AChE expression was largely positive around the tricuspid ring and dispersed in distribution (arrow). (C) Cx43 (green) and HCN4 (red) double-staining showed reciprocal Cx43 and HCN4 expression. (D) Cx40 (green) and Cx45 (red) double-label staining showed abundant Cx45 expression in the penetrating bundle $(\mathrm{PB})$ tissue and areas along the atrial side where $\mathrm{Cx} 43$ expression was absent. Cx40 was expressed only in the PB tissue. (E) Masson's trichrome-stained section. (F) AChE staining: Rusty-colored tissues highlighted a linear connection with the atrioventricular node (AVN) from the atrial side. (G) Cx43 (green) and HCN4 (red) double-staining revealed a correlation with AChE staining. (H) Cx40 (green) and Cx45 (red) double-staining showed abundant Cx45 expression in areas without $\mathrm{Cx} 43$ expression. PB tissue showed a longitudinal dissociation, with the volume of $\mathrm{Cx} 40$ expression decreasing compared with that at the former level (elliptical area). Cx40 expression was not present on the atrial side. (I) Masson's trichrome staining showed the inferior extension of the AVN located on the atrial side above the tricuspid annules. $(\mathrm{J})$ AChE staining was limited to the INE area. (K) Cx43 expression was absent in the inferior extension where HCN4 expression was present. The inferior extension divided into the right extension and the left extension (arrow). (L) Cx45 expression was more abundant in the INE than in other atrial areas (a slight fold in the section). No Cx40 expression was observed in the inferior extension.

Figure $\mathrm{B}(\mathrm{a})$ shows the location of images shown in Figures $\mathrm{C}(\mathrm{a})$ and $\mathrm{D}(\mathrm{a})$, while Figure $\mathrm{B}(\mathrm{b})$ shows the location of images shown in Figures $\mathrm{C}(\mathrm{b})$ and $\mathrm{D}(\mathrm{b})$. Figure $\mathrm{F}(\mathrm{a})$ shows the location of images shown in Figures G(a) and 2H(a), while Figure F(b) shows the location of images shown in Figures G(b) and H(b).

Figure 3. Continuous sections at the level of start of the $\mathrm{CN}$ and the end of the RN from another sample. (A1-A3) Part of the node-like tissue was separated from the RN and penetrated into the fibrous rings between the atrium and ventricle (dotted box). (B1-B3) The following continuous sections showed that the nodal tissue continued in the fibrous rings. (C1-C3) The following continuous sections showed that the nodal tissue descended into the CN. (A3, B3, C3) HCN4 labeling further suggested that the penetrated tissue was node-like tissue (dotted box). (D-F) The region connecting the RN and CN was also near the start of the tendon of Todaro (black arrow). Two narrow connections between the RN and $\mathrm{CN}$ at different levels near the 
tendon of Todaro (dotted box). After the node-like tissue descended into the CN, fibrous tissue surrounding the other part of the $\mathrm{CN}$ seemed to have no direct connection with the atrium.

Figure 4. (Left) Eighty model sections. The distance between groups is $6 * 4=12 \mu \mathrm{m}$. (Right) Anatomical model views of the rat atrioventricular node $(\mathrm{AVN})$. (A and B) Nodal tissue model viewed from the right and left. (C and D) Nodal (yellow) and penetrating bundle (red) tissue models viewed from the right and left. (E and F) Conduction system tissue and valve ring (blue) model viewed from the right and left.

Figure 5. (A) $\mathrm{Na}_{\mathrm{v}} 1.5$ expression was abundant in the atrial and ventricular muscles but weak in the PB and RN. Ca 3.1 was mainly expressed in the PB and RN. (B) and (C) The CN and INE also weakly expressed $\mathrm{Na}_{\mathrm{v}} 1.5$ but positively expressed $\mathrm{Ca}_{\mathrm{v}} 3.1$. (D) Analysis of the $\mathrm{Na}_{\mathrm{v}} 1.5$ immunofluorescence staining intensity showed that the RN, $\mathrm{CN}$ and INE all presented significantly low $\mathrm{Na}_{\mathrm{v}} 1.5$ expression $(P<0.001)$. No significant differences in expression were observed among the RN, CN and INE $(P=0.104)$, but a trend toward slightly higher $\mathrm{Na}_{\mathrm{v}} 1.5$ expression in the $\mathrm{RN}$ than in the other two regions was observed.

Figure 6. Hypothesis of the structure and functional relationship of the AVN. (A) During sinus rhythm, the action potential propagates preferentially along the RN due to the presence of fewer connective tissue barriers to the atrial tissue. (B) In the slow-fast form of AVNRT, anterograde conduction occurs through INE inputs, and retrograde conduction occurs through the RN. Multiple breakthroughs of atrial activation occur during $\mathrm{A}-\mathrm{V}$ nodal reentry due to the dispersed distribution of the RN.

\section{Figures}




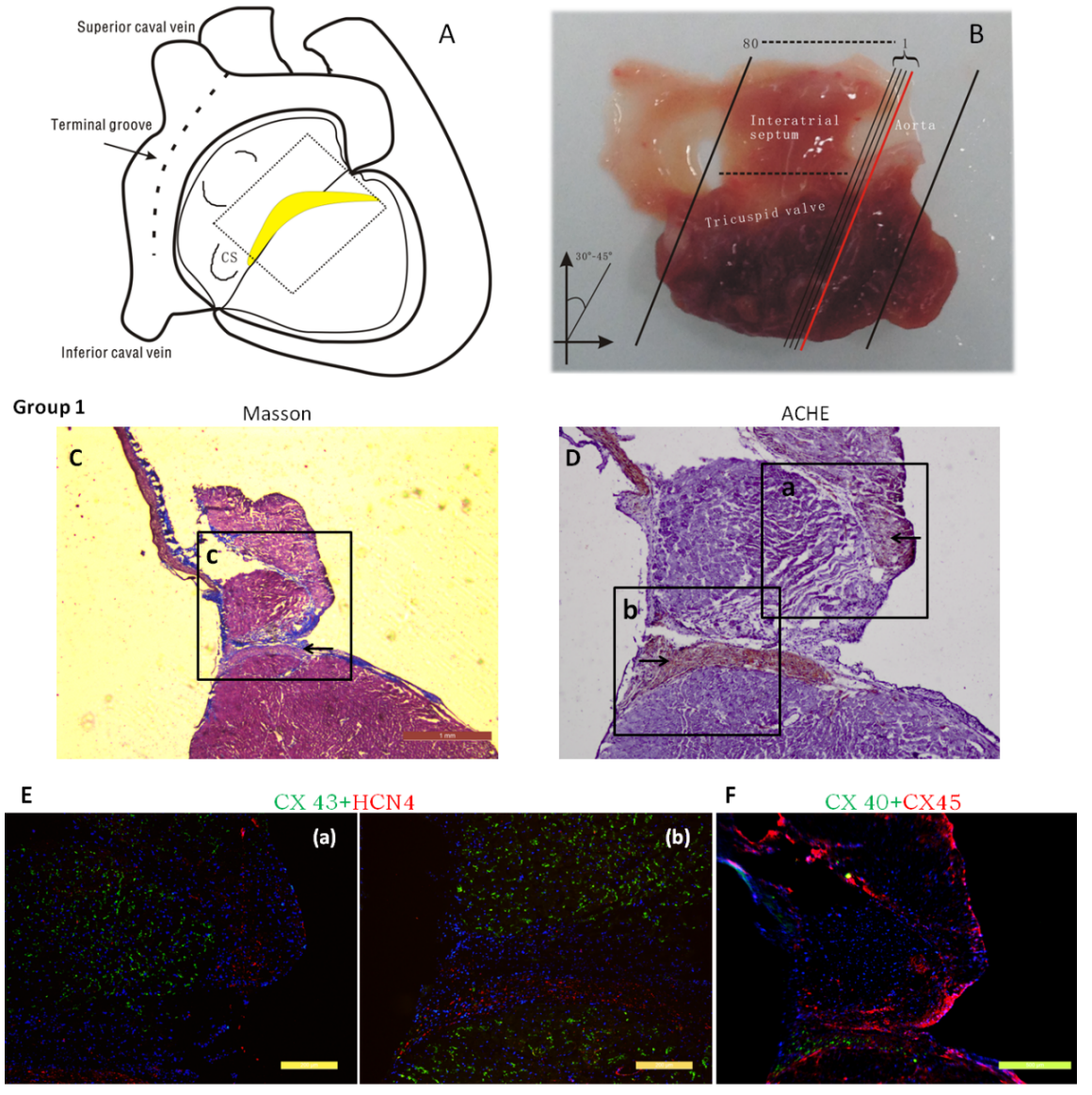

Figure 1 


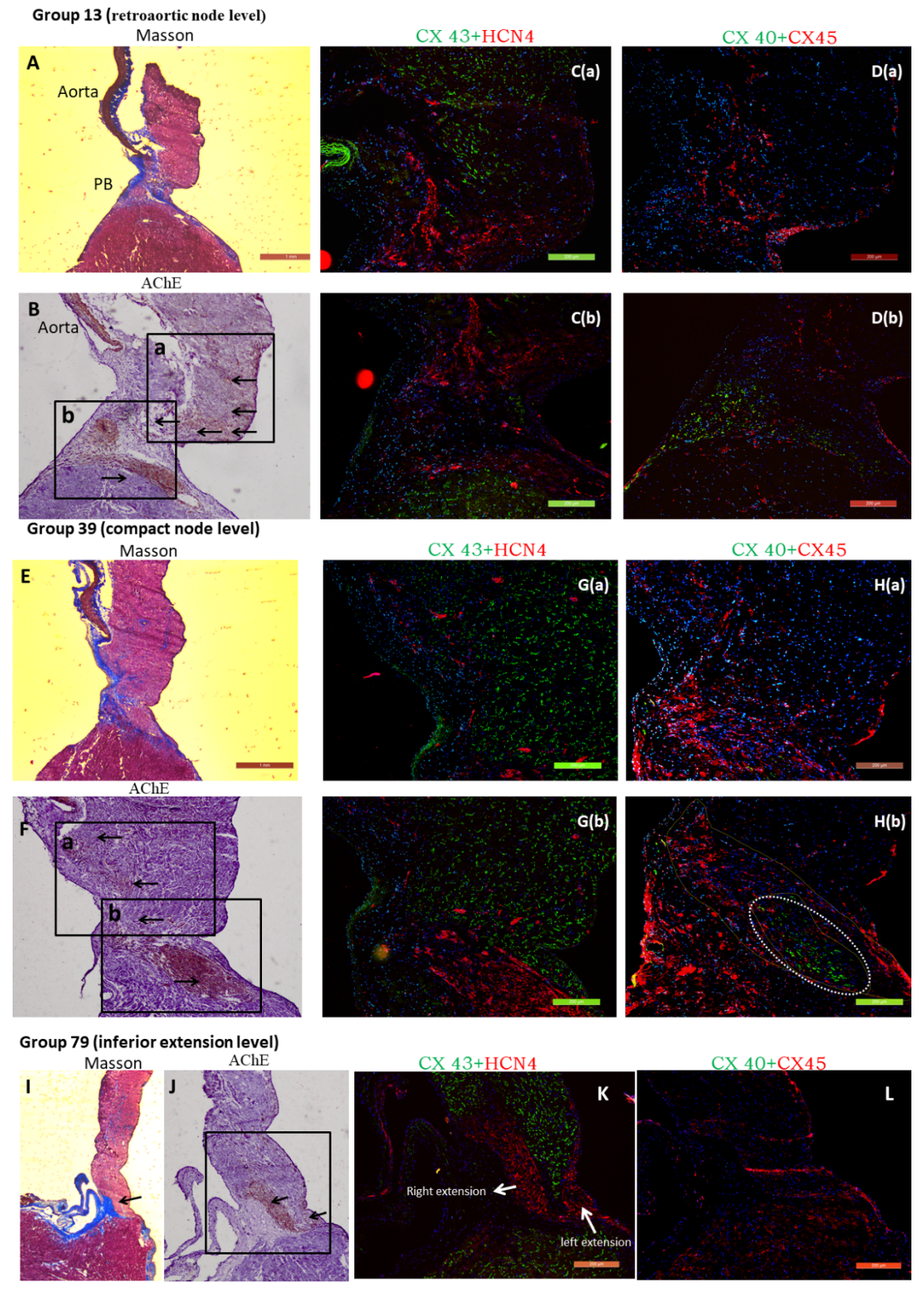

Figure 2 


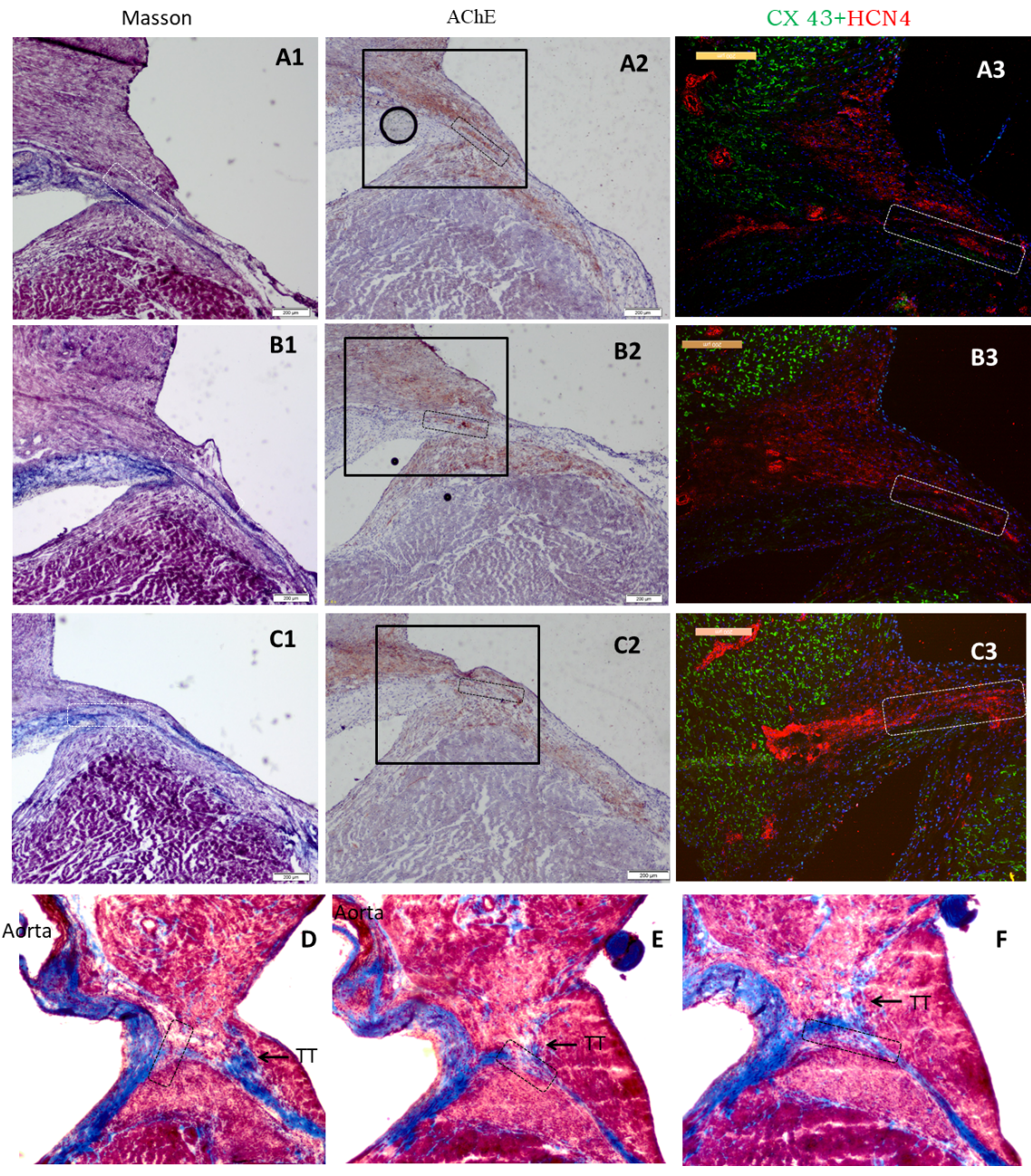

Figure 3 


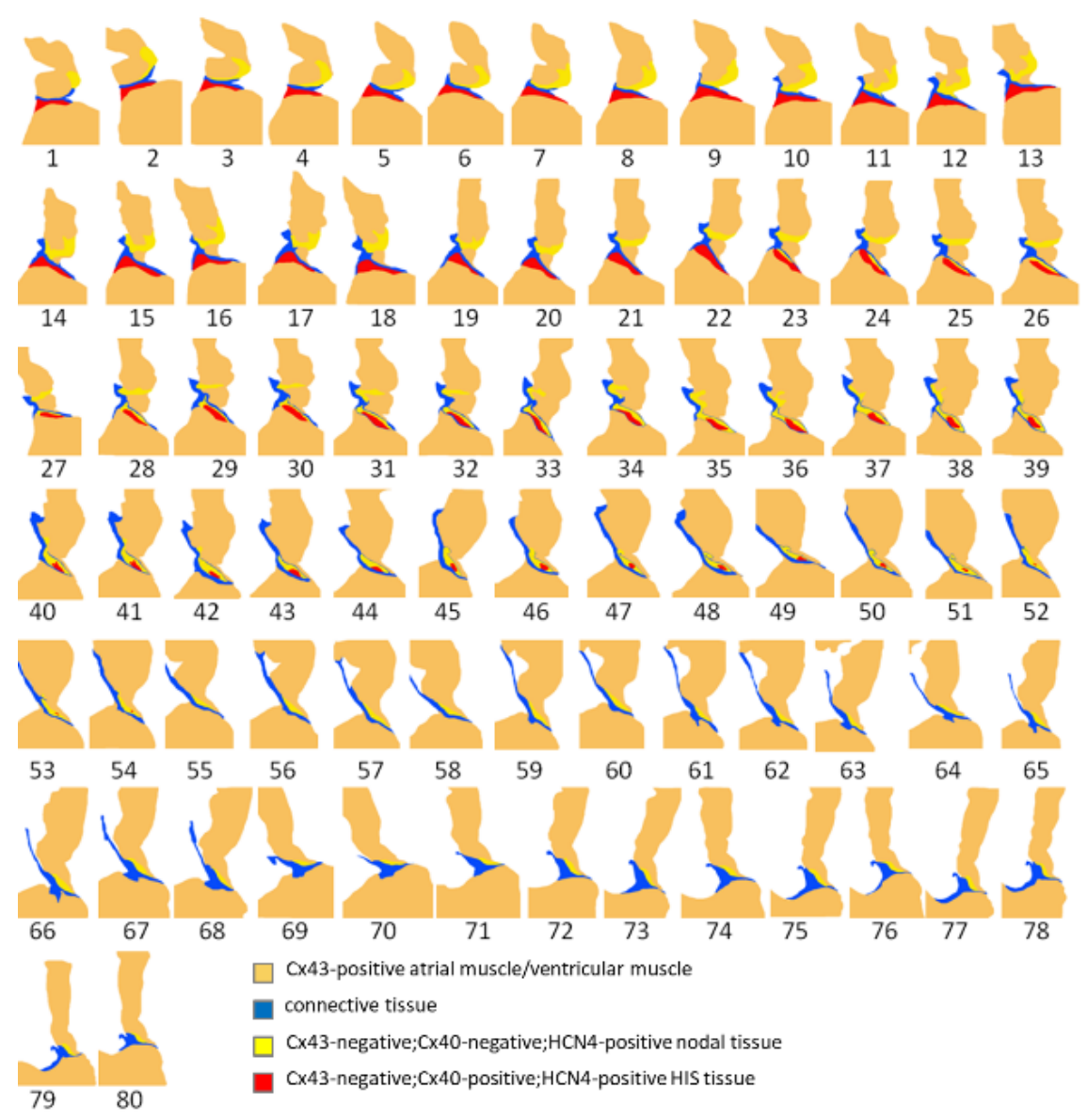




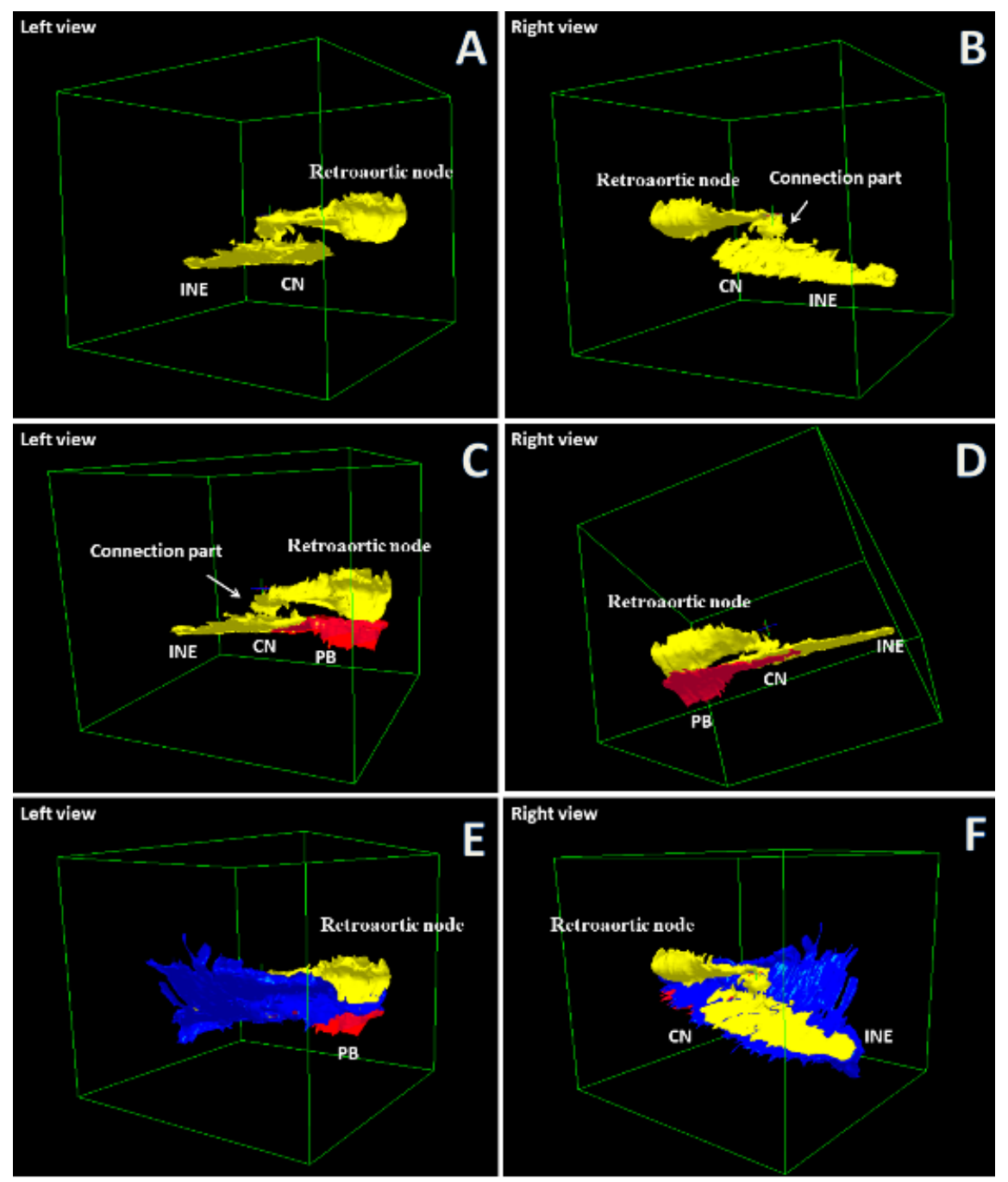

Figure 4 

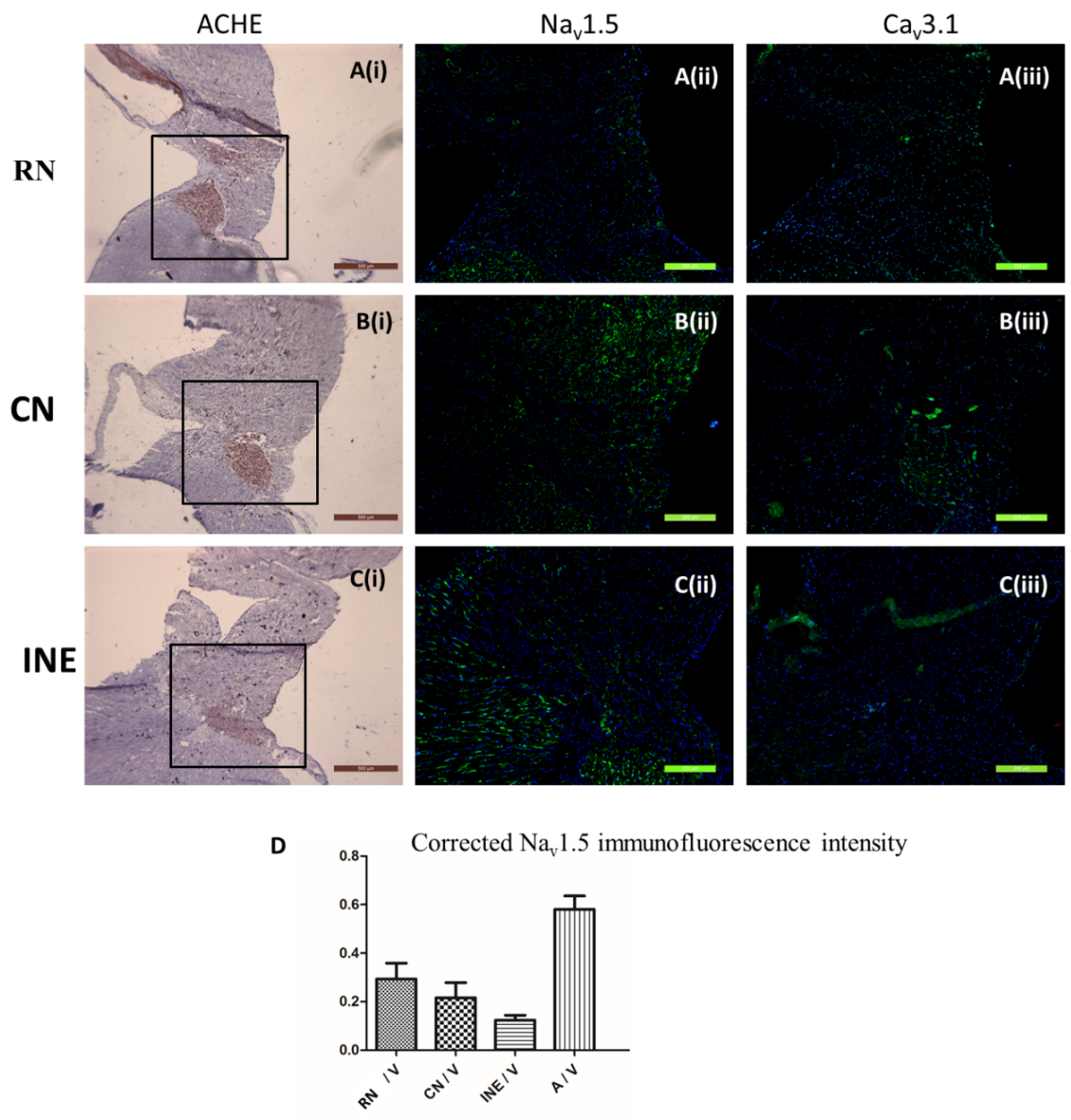

Figure 5 

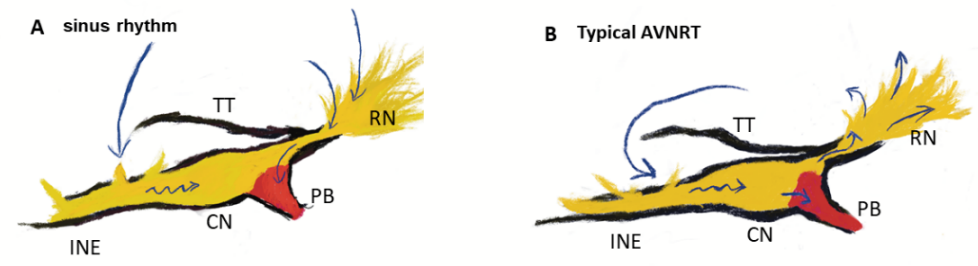

Figure 6 